\title{
Ronan CHALMIN, Lumières et corruption
}

Paris, H. Champion, 2010

\section{Christian Albertan}

\section{(2) OpenEdition}

\section{Journals}

Édition électronique

URL : https://journals.openedition.org/ahrf/13212

DOI : 10.4000/ahrf.13212

ISSN : 1952-403X

Éditeur :

Armand Colin, Société des études robespierristes

\section{Édition imprimée}

Date de publication : 1 juin 2014

Pagination : 203-204

ISBN : 978-2-908327-68-7

ISSN : 0003-4436

\section{Référence électronique}

Christian Albertan, «Ronan CHALmin, Lumières et corruption », Annales historiques de la Révolution française [En ligne], 376 | avril-juin 2014, mis en ligne le 11 juillet 2014, consulté le 01 juillet 2021. URL : http://journals.openedition.org/ahrf/13212 ; DOI : https://doi.org/10.4000/ahrf.13212

Ce document a été généré automatiquement le 1 juillet 2021

Tous droits réservés 


\title{
Ronan CHALMIN, Lumières et corruption
}

Paris, H. Champion, 2010

\author{
Christian Albertan
}

\section{RÉFÉRENCE}

Ronan CHALMIN, Lumières et corruption. Paris, H. Champion, 2010, 388 p., ISBN

978-2-7453-2030-8., $88 €$.

1 Le XVIII ${ }^{e}$ siècle est usuellement associé à l'idée d'optimisme, de progrès, de bonheur. Les Lumières ouvriraient les perspectives exaltantes d'un avancement indéfini de l'humanité progressivement débarrassée des obscurités du fanatisme et de la routine. C'est oublier que les notions de progrès et de bonheur ne résument pas à elles seule cette période singulièrement riche et complexe, peut-être même contradictoire dans ses goûts et ses centres d'intérêt: le siècle des Lumières a assurément aussi ses obscurités, ses versants sombres. C'est ce qu'entreprend de démontrer Ronan Chalmin dans cette étude stimulante sur les liens entre la notion de corruption et la pensée du XVIII ${ }^{e}$ siècle français.

2 En s'appuyant sur l'œuvre de six auteurs importants du siècle des Lumières ayant écrit sur des sujets et dans des genres fort différents (Crébillon, Diderot, Montesquieu, Rousseau, Robespierre et Sade), l'auteur fait ressortir la place singulièrement importante occupée par l'idée de corruption dans la pensée du XVIII siècle. Selon lui, «toute instance critique à l'âge des Lumières a pour fondement » la mise en accusation $\mathrm{du}$ monde convaincu de corruption. Cette dernière participe du développement du monde et annonce en même temps la fin de celui-ci. Pour les auteurs du siècle des Lumières, il n'y aurait pas en définitive de civilisation sans corruption et vice versa.

3 L'auteur invoque naturellement en premier lieu Montesquieu, dont trois œuvres majeures au moins font une large place à l'idée de corruption. Les Considérations sur les causes de la grandeur des Romains et de leur décadence, l'Esprit des Lois et certains passages des Lettres persanes contiennent de fait une réflexion poussée sur la corruption en politique et dans le domaine social. Avec lui, la corruption deviendrait même une sorte 
de loi historique à l'œuvre à toutes les époques et dans toutes les sociétés : il serait dans l'ordre des choses que les constructions politiques et sociales se corrompent et aillent, en évoluant dans le temps, vers leur fin. L'auteur trouve d'autres arguments en faveur de sa thèse dans l'œuvre de Jean-Jacques Rousseau, dans laquelle la corruption joue effectivement un rôle important. L'homme en société n'est pour Rousseau que le produit d'une corruption qui dès le départ l'a éloigné, pour son malheur, de l'état de nature, la civilisation et la culture n'étant qu'un long procès de corruption. Cette dernière est aussi un des fondements de la pensée politique de Robespierre, l'« Incorruptible », qui se réclame clairement de Jean-Jacques.

4 Mais la pensée de la corruption ne caractérise pas que le début et la fin du siècle: l'auteur met en exergue l'importance accordée à l'idée de corruption dans l'œuvre de deux écrivains du milieu du siècle, Crébillon fils et Diderot. Le premier, comme nombre d'auteurs de romans libertins, fait coexister dans ses romans l'idéal de pureté et les mœurs corrompus, le second entreprend de mettre le savoir à l'abri de la corruption en se lançant dans la grande entreprise encyclopédique. À y regarder de plus près, la notion de corruption joue également un rôle central chez Sade. Mais ici, la notion cesse d'être négative : chez le divin marquis, toujours selon l'auteur, la corruption n'est pas la source des maux, le signe d'une décadence, la manifestation d'un mal à combattre. Elle conditionne l'accès au plaisir et à la vérité. C'est en définitive un autre XVIII ${ }^{\mathrm{e}}$ siècle que nous invite à lire l'auteur, un siècle des Lumières travaillé par un certain pessimisme.

5 Avec cet ouvrage, Ronan Chalmin nous livre un essai stimulant, plaisant à lire et reposant sur une information étendue (références nombreuses à la pensée de saint Augustin, mais aussi à des philosophes et critiques de notre temps comme Blanchot, Valéry, Serres, Morin, Derrida). Les littéraires, en faisant abstraction de quelques néologismes (ex. le «masquage » pour le fait de masquer), tics et approximations de langue (qu'est-ce que la "grammaire libertine»?), goûteront sans doute plusieurs passages et certaines analyses stylistiques. Les historiens des idées seront sans doute plus réservés à l'égard de ce travail, certes original et piquant, mais aventureux sur plus d'un point. L'auteur, entraîné par son hypothèse, lit souvent, en effet, de manière fort personnelle et intéressée les auteurs qu'il étudie.

6 C'est arbitrairement et sans rien véritablement démontrer qu'il situe en 1750 un « changement radical » dans la conception du savoir (op. cit., p. 119). Y a-t-il autour de cette date, et non pas précisément cette année-là, basculement ou début d'une série de faits conduisant à un changement dans l'ordre du savoir? Autrement dit, y a-t-il rupture ou glissement, évolution de la conception de ce qu'est le savoir? Est-ce encore parce qu'il serait un "penseur bourgeois » que Diderot se ferait encyclopédiste en transformant le grand dictionnaire en "coffre-fort» du savoir (op.cit., p.129)? L'auteur se donne également des facilités lorsqu'il aborde certains faits et se montre même à l'occasion fort négligent. Qu'est-ce précisément que ce "discours " que le (jeune) Turgot prononce en Sorbonne en 1750 (op. cit., p. 120) ? S'agit-il de sa thèse ? Et en quoi ce « discours » est-il révélateur de ce qui se pense alors dans Paris? L'auteur a manifestement une connaissance superficielle du fonctionnement de la Sorbonne et des débats d'idées des années cinquante... D'autres questions se posent. Peut-on, par exemple, mettre sur le même plan toutes les formes de corruptions ? Observons encore que l'économie générale de l'essai de Ronan Chalmin est déconcertante : un quart de l'ouvrage est consacré... à Robespierre, qui n'est pas à proprement parler un représentant des Lumières. Si on ajoute à cette interminable partie consacrée à 
Robespierre celle qui porte sur Sade, on arrive à la moitié de l'ouvrage. C'est beaucoup pour l'extrême fin du siècle et cela ouvre des béances dans le reste de la période. À l'évidence, Ronan Chalmin nous entretient dans ce livre de ses passions et de ses hypothèses, souvent très personnelles. Il soulève au passage des questions intéressantes et dérangeantes, mais son essai ne nous fera pas oublier les travaux fondateurs et auxquels on ne cesse de revenir de Robert Mauzi, de Jean Ehrard et de Jean Deprun sur l'idée de bonheur, de nature ou encore sur l'idée d'inquiétude au siècle des Lumières. 